\title{
Pseudo-Scalar Current and Weak Type Interaction in Fourth Generation Quarks Models
}

\author{
Aayush Verma* \\ Email: aayushverma6380@gmail.com
}

In this note, the toy-model of Fourth Generation quarks coupling to Standard Model particles through unknown Lagrangian has been figured. Eventually producing a $10^{3} \mathrm{TeV}$ vector gauge boson, which is a special gauge boson that appears in unification models and here as a mediator for WeakSM4 interaction. Another anomaly of different gauge boson has been reported.

Keywords Beyond the Standard Model; Gauge Sector

\section{INTRODUCTION}

In the last note by the author [1, it was shown that there may be a current which is responsible for symmetry breaking and acquiring a gauge boson of $10^{3} \mathrm{TeV}$, which was laid on the work of Quinn, Georgi, and Weinberg [2]. Here we will try to figure out the significance of this boson and other phenomenology in SM4 [3].

\section{PSEUDO-SCALAR CURRENT AND INTERACTION}

We start with describing the Lagrangian of interaction between SM4 and the current model of SM,

$$
\mathcal{L}=\alpha+\zeta_{c}
$$

in the last note [1], we defined the $\zeta_{c}{ }^{1}$ as the current. But let us go to more fundamental level and inquire the role of this $\zeta_{c}$.

We write the Lagrangian of altered QCD gauge group and current SM gauge theory as [4,

$$
\mathcal{L}=S O(2) \times S U(3) \times U(1) \times S U(3+\beta)
$$

where $\beta$ is different choices. but here we will set $\beta=2$, evidently making it another GUT. So the Lagrangian describing the overall interaction between SM4, Quarks in particular, and SM. It can be noted that if this interaction does happen, which we can hope to find using perturbation, then interaction must have produced some scales and other particles, for sure. Let us say, the interaction and excitation in the field gained by overall in Eq. 2 give us a light mass particle, let us say $\Delta_{S M_{4 \times 3}}{ }^{2}$, through the analysis [3]. There may be many particles, but we opt it for convenience, which is by no means connected to Delta Baryon.

\footnotetext{
* Jeewan Public School, Motihari, 845401, India

1 Under-script "C" has been written to clear the confusion, it means mathematical current, not the physical current.

2 We don't need to know the particular state or even which categorization it belongs, because it is a virtual particle
}

The field theory of $\Delta_{S M_{4 \times 3}}$ denote as $\phi\left(\Delta_{S M_{4 \times 3}}\right)$, which symmetry spontaneously break. As per this the Lagrangian can be introduced as,

$$
\mathcal{L}=H_{n}+\zeta_{c}
$$

where $H_{n}$ is $\alpha$ of Eq. 1, which describes the Higgs Mechanism [5] and $\zeta_{c}$ the introduced current. We make this current out of $\Delta_{S M_{4 \times 3}}$, because we believe that $\Delta_{S M_{4 \times 3}}$ has some $\gamma^{\alpha}$ mathematics, which is carried by the $\zeta_{c}$ part.

We can hope that $\zeta_{c} \rightarrow \gamma^{\alpha} \lambda$ or $\zeta_{c} \rightarrow \gamma^{\alpha} C$, where by convention it don't need any $\gamma^{\mu}$. Where $\lambda$ is an adjustable parameter and $\mathrm{C}$ is a constant and $\alpha$ is a number that produces the pseudo-scalar object. But this is not any mechanism related to Electroweak, but it is an isolated mechanism, may be an effective field. However, this $\zeta_{c}$ is not an analogy of $1-\gamma^{5}$.

This tells us that, this induced current from $\phi\left(\Delta_{S M_{4 \times 3}}\right)$ which comes from the interaction of Eq. 2 leads to a pseudo-current in SM4.

Now the $\zeta_{c}$ which by using Yukawa Couplings makes interaction to SM (Current Standard Model),

$$
\mathcal{L}_{\zeta_{c} \times S M 3}=\zeta_{c} \times S O(2) \times S U(3) \times U(1)
$$

which leads to

$$
\gamma^{\alpha} \lambda / C(S O(2) \times S U(3) \times U(1))
$$

where $\lambda / C$ is our answer to an imperfect question that whether the parameter is adjustable or constant, but a parameter is needed.

By a simple analysis and making the interaction between only $\zeta_{c}$ and Weak field makes up a superimposed current,

$$
\gamma^{\alpha} \lambda / C\left(1-\gamma^{\alpha}\right)=\lambda / C\left(\gamma^{\alpha}-\gamma^{\alpha} \gamma^{5}\right)
$$

which we forsake should be calling "Weak-SM4 $\zeta_{c}$ ". Now this Weak-SM4 $\zeta_{c}$ (WSC) is the type of vectorial/scalar (because we don't have the idea of $\alpha$ ) object in the formalism and theory of interaction between only SM4 and Weak, by weak, we mean coupling of SM4 to other particles through the weak force. 


\section{THE GAUGE BOSON}

According to the previous note [1], either $\zeta_{c}$ or WSC is the reason for no interaction between particles and SM4 particles. But we came to know, interactions do happen but only by the weak medium.

But we don't see any interaction happening between SM4 and SM particles. There can be 3 cases to explain this: 1) It may be a possibility that this WSC requires only heavy particles to interact. 2) There may be another type of gauge boson for interaction. 3) A modified version of 1 , maybe this current only produces at the only high energy level.

If it is 1 , then there must be other heavy particles in SM. If it is 2, which I believe is, which perhaps was shown in [3] correct. If 3 , then what are the modified STU parameters for this?

All three may be correct, because t' and b' which are quarks of SM4 are at most of lower bound $>4$ $\mathrm{Tev}$ and $>1.3 \mathrm{TeV}$ respectively [3]. And the gauge boson we are talking about is, perhaps the acquired mass boson from Higgs mechanism of Eq. 3 of $10^{3}$ $\mathrm{TeV}[2]$ [6]. So this may be a special kind of gauge boson in Weak mechanism besides $\mathrm{W}$ and $\mathrm{Z}$, many times the mass of $\mathrm{W}$ and $\mathrm{Z}$ boson. They could be a boson for Weak-SM4 interaction, which perhaps nothing but a vectorial gauge boson of $\mathrm{SU}(5)$ which we have taken in Eq. 2.

WSC or $\zeta_{c}$ is then something new, which is not thought about. But we should because we have faced already one kind of current issue. It is evident that in Eq. 5 , the $\zeta$ doesn't interact with any other gauge group. And because $\phi\left(\Delta_{S M_{4 \times 3}}\right)$ is an excitation of SM4 and SM interaction, we led to believe that SM4 particles don't interact with ordinary matter. Even if they by SM4, they may require some heavy particles, like top quark. But we haven't seen any event of such kind. If every theory have pseudoscalar current and have a heavy mass particle, interaction is possible.

Adjustable Parameter of $\zeta$ may play an important role in determining more about this, but if there is a Constant, then it will produce another question about the constant. But one thing is clear that interaction to happen between SM4 requires a heavy mass gauge boson.

There may be a version of Weak-SM4(Leptons) interaction, but there is an important constraint on these. But we eventually see that $\zeta$ is an important part of SM4 and one should not overlook these.

\section{CONCLUSION}

It is now almost clear, but in this model, that there may be some special kind of interactions between fourth-generation particles and other particles through the Weak Force. This is mediated by an updated boson of much bigger mass than W and Z. Having a mass of $\mathrm{TeVs}$ scales, this boson comes from the unified theory.

[1] A. Verma 10.20944/preprints202008.0191.v1 (2020).

[2] H. Georgi, H. R. Quinn, and S. Weinberg, in Origin Of Symmetries (World Scientific, 1991) pp. 334-337.

[3] A. Verma, Revisiting constraints of fourth-generation quarks and leptons (2020).

[4] H. Georgi and S. L. Glashow, Physical Review Letters 32, 438 (1974).

[5] P. W. Higgs, Physical Review Letters 13, 508 (1964).

[6] J. Goldstone, A. Salam, and S. Weinberg, Physical Review 127, 965 (1962). 